\title{
CENTRO DE DESARROLLO PARA LA PEQUEÑA EMPRESA. REVISIÓN SISTEMÁTICA DE LA LITERATURA
}

\author{
SMALL BUSINESS DEVELOPMENT CENTER. SYSTEMATIC \\ LITERATURE REVIEW
}

\section{Gioconda Vargas-Morúa ${ }^{1}$}

\section{RESUMEN}

El propósito de este artículo es estudiar científicamente la actividad y los alcances de los Centros para el Desarrollo de la Pequeña Empresa (SBDC), a través de una revisión sistemática de la literatura. Para tal fin, se realiza una búsqueda booleana y se extrae la información de tres bases de datos: Scopus, SSCI y EBSCOhost. Los objetivos específicos son: identificar las diferentes perspectivas teóricas que caracterizan y definen la literatura relacionada con los SBDC y destacar las conexiones entre ellas; determinar la perspectiva a la que los investigadores le han otorgado mayor importancia de acuerdo con un análisis de tendencias de las publicaciones; identificar los mayores exponentes del tema e identificar las fuentes que mayormente son consultadas. Para obtener la información bibliométrica se utilizaron técnicas de citación para seleccionar, analizar e interpretar los patrones dentro de la literatura. Se logra: identificar el desarrollo de negocios y la educación empresarial como las dos principales perspectivas teóricas; se establece la educación empresarial como la perspectiva de mayor importancia dado su crecimiento; se reconocen los mayores exponentes, $y$ fuentes que publican el tema. Desde un aspecto práctico, esta investigación contribuye al análisis de la teoría existente sobre SBDC y evidencia la efectividad de estos.

PALABRAS CLAVE: ESTRATEGIAS DE DESARROLLO; FORMACIÓN; CENTRO DE ENTRENAMIENTO; UNIVERSIDADES.

CLASIFICACIÓN JEL: O1, O170, M2, M20, M21

\section{ABSTRACT}

The purpose of this research is to show how advisory and preventive services are approached by the Internal Audit Units (IAU), which is carried out under a quantitative and descriptive methodological approach, and is based on the results selected from a questionnaire of 50

1 Universidad Estatal a Distancia, Observatorio de la Micro, Pequeña y Mediana Empresa (OMiPYME); Código Postal: 11502; San José, Costa Rica; gvargasm@uned.ac.cr 
questions, divided into the following categories of analysis that support the research: UAI methodologies used to provide warning services and preventive advice; utilities of this type of services; and difficulties that arise to be able to carry them out; applied to heads and collaborating technicians of $22 \mathrm{UAI}$ of the Costa Rican public sector. The results obtained show that, for the most part, a management instrument is used to carry out these services. In addition, requests for advice or warnings that arise and are issued in accordance with the Costa Rican legal and technical system are permanently attended to.

KEYWORDS: DEVELOPMENT STRATEGIES; TRAINING; TRAINING CENTER; UNIVERSITIES.

JEL CLASSIFICATION: 01, 0170, M2, M20, M21

\section{INTRODUCCIÓN}

Los Centros de Desarrollo Empresarial nacen como una iniciativa de apoyo técnico, aprendizaje permanente, innovación y transformación de los sistemas económicos locales en los países europeos, en la década de los '70. Posteriormente, en la década de los ' 80 e inspirados por los Small Business Development Center de Estados Unidos, los países latinoamericanos desarrollan los propios centros-por lo que se hará referencia a ellos por sus siglas en inglés SBDC. Actualmente, son muchos los países de América Latina que han ajustado los SBDC a su idiosincrasia, a sus instituciones y a su economía; a fin de apoyar positivamente a las micro, pequeñas y mediana empresas (mipymes) (Dini, 2019).

Algunas características que los SBDC tienen en común es que son un intento por institucionalizar la acción de apoyo a las mipymes con proyección al mediano y largo plazo; es un modelo de gestión orientado a los resultados y evaluación permanente de los impactos generados por las empresas atendidas; se desarrollan a partir del esfuerzo conjunto de distintos sectores de la sociedad: sector público, académico y empresarial, que colaboran aportando recursos y conocimiento para el crecimiento de las mipymes; la modalidad de atención es integral y asistida a lo largo de todo el proceso de transformación; $y$ por último operan de manera descentralizada lo que permite que se adapten al territorio en que se localizan (Dini, 2019).

Por lo tanto, en este momento de pandemia Covid 19-, en que los gobiernos buscan reactivar sus economías de manera sostenida y coordinada, el modelo SBDC se convierte en el medio propicio para que las mipymes se reestructuren, se digitalicen, logren visualizar las nuevas oportunidades y se reinventen, se vuelvan eficientes, $y$ logren el objetivo de crecimiento económico esperado.

Siendo así, las universidades como instituciones educativas aportan a los SBDC conocimiento y asesoría en el proceso emprendedor, pues deben motivar del uso eficiente de los recursos, impulsar la toma de decisiones, visualizar oportunidades, mostrar y orientar nuevas rutas de éxito para las empresas, entre otros aspectos.

El informe Global Entrepreneurship Monitor (GEM) indica que cada economía tiene su propio perfil empresarial específico y vive un proceso empresarial propio, por lo que cuando se habla del índice de actividad emprendedora total (TEA -siglas en inglés) se deben tener en cuenta las diferentes coyunturas. Sin embargo, en términos generales se sabe que antes de la pandemia, el nivel de crecimiento de las iniciativas emprendedoras a nivel mundial iba en aumento (Bosma \& Kelley, 2019), y que los SBDC no solo formaban parte de las políticas de apoyo a las mipymes, sino que también contribuían al sistema productivo en el cual se insertaban (Dini, 2019), por lo que es de esperar que las SBDC trabajen como promotoras de la reactivación de las economías.

Adquirir conocimientos de los SBDC y sus alcances, es un desafío que se abordará en este documento en pro del beneficio del país, de los investigadores y del sector empresarial. El fin último es contribuir en la investigación de los SBDC a través de una revisión sistemática de la literatura y que posteriormente los emprendedores puedan definir nuevas estrategias que les permitan la estabilidad y a nivel de país se dé un crecimiento de la economía. 
Por tanto, este documento contribuye a la investigación en empresa en dos aspectos muy importantes: el primero es que es el primer estudio sistemático de la literatura que se realiza sobre el tema de SBDC y recaba la literatura científica internacional de tres bases de datos. La segunda, es que esta revisión, que muestra el universo de información existente alrededor de los SBDC, también identifica el efecto que los SBDC han logrado en las iniciativas emprendedoras a fin de que estas se fortalezcan al adquirir capacitaciones y apoyo para reinventarse. Es decir, señala a nivel de educación y negocios, las buenas prácticas para el desarrollo de las mipymes que, en este tiempo de pandemia, pueden constituirse como el punto decisivo para permitir la subsistencia y posterior desarrollo de estas.

Dados los puntos anteriores, se reconoce que las investigaciones que hasta ahora se han encontrado sobre SBDC proporcionan parte del fundamento científico que se debe seguir construyendo, por lo que determinar aquellos aspectos que han sido mayor o menormente tratados, permitirá visualizar las futuras investigaciones $y$, por lo tanto, subsanar las lagunas en este campo científico.

Para la búsqueda de la literatura se utilizaron las siguientes bases de datos: Thompson Reuters' Social Science Citation Index (SSCI), Elsevier's Scopus y EBSCOhost. Es importante mencionar que, aunque existe mucha información al respecto, no toda cumple con la condición de ser científica, arbitrada y específicamente relacionada con los SBDC, por lo que esta búsqueda no se limita a un tiempo determinado, sino que se considera toda la información que contienen las bases de datos.

En esta investigación se recopilen datos bibliográficos y se analiza la información con el objetivo principal de estudiar científicamente la actividad y los alcances de los SBDC. Para alcanzar dicho objetivo se establecen los siguientes objetivos específicos:

- Identificar las diferentes perspectivas teóricas que caracterizan y definen la literatura relacionada con los SBDC y destacar las conexiones entre ellas.

- Determinar la perspectiva a la que los investigadores le han otorgado mayor importancia de acuerdo con un análisis de tendencias de las publicaciones.

- Identificar los mayores exponentes del tema a través del número de citas en los artículos asociados con los SBDC.

- Identificar las fuentes que mayormente son consultadas sobre los SBDC.

Se buscó principalmente que la calidad de la información fuera óptima y se pudiera responder a las preguntas de investigación: ¿En qué áreas los SBDC adquieren mayor relevancia?, ¿cuál ha sido su importancia a través del tiempo?, ¿Cuáles son los autores mayormente citados? y ¿Cuáles son las fuentes que mayormente son consultadas?

En este sentido, en esta investigación integra la información que se encontró y se agrupa de acuerdo con las diferentes perspectivas que tratan el tema de SBDC.

\section{MARCO TEÓRICO}

Los centros de desarrollo empresarial (CDE) surgen en Europa al comienzo del siglo veinte. Inicialmente, son impulsados por la cámara de comercio y en la década de los 80' se emplean como instrumentos de política pública para el apoyo a las mipymes (Dini, 2019) por la importancia que tienen en el desarrollo económico de los países.

De acuerdo con la Comisión Económica para América Latina y el Caribe (CEPAL) algunas aspectos que otorgan importancia y caracterizan las mipymes latinoamericanas, a diferencia de los países industrializados, es que son responsables de aproximadamente dos tercios de los empleos 
formales; generan alrededor del 75\% de la producción (Dini, 2019); representan aproximadamente el 99\% de las empresas en América Latina (Dini \& Stumpo, 2018), entre otros.

Por tanto, las mipymes representan el tema central del desarrollo de la región y el punto álgido en el desarrollo de políticas que brinden respuestas a la problemática social, pues ha existido una descoordinación entre las entidades de fomento y la escasa integración de políticas nacionales de trasformación productiva, que han impedido una adecuada formalización de los esfuerzos (Dini, 2019) dejando como saldo trabajadores con salarios bajos, familias pobres y economías lentas.

El concepto de CDE que propuso la Comisión Europea es la siguiente "los CDE son aquellos servicios generados por una iniciativa pública, que apuntan a asistir a las empresas o a los empresarios, para que puedan desarrollar exitosamente sus negocios y responder eficazmente a los desafíos que su ambiente físico, social y de negocio les plantea" (Dini, 2019, p. 14). Por tanto, esta modalidad se concentra en la prestación de soporte profesional y acompañamiento para la transmisión de nuevos conocimientos y el desarrollo de nuevas capacidades en las empresas.

Las empresas buscarán acceder a este tipo de soporte profesional o acompañamiento a fin de obtener conocimientos y desarrollar las capacidades empresariales, que de otra manera no obtendrían, pues los servicios tienen costos elevados o se requiere de consultas conjuntas que impiden el acceso de las empresas. También, las empresas buscarán contactar con los CDE, a fin de estructurar y establecer estrategias; adquirir conocimiento en tecnología; desarrollar competencias, entre otros (Dini, 2019).

Por su lado, en Estados Unidos, en el año 1976, nacen los Small Business Development Center (SBDC) como una iniciativa de la Universidad Politécnica del Estado de California, y posteriormente se constituyó la red de centros. Para el año 2000, inicia una labor de difusión del modelo en los países Latinoamericanos bajo el apoyo del Banco Interamericano de Desarrollo (BID) (Dini, 2019).

En la actualidad, en América latina hay numerosas instituciones de fomento que han impulsado la creación de los centros de desarrollo, inspirados en los SBDC y cuyas características tienen los siguientes elementos: la gestión y financiamiento basados en la colaboración entre el gobierno, la academia y el sector privado; acción orientada a los resultados y registro constante de las mejoras generadas por la acción de los centros en las empresas atendidas, en particular en cuanto a generación de empleo, incremento de ventas y acceso a crédito; asistencia a largo plazo (Dini, 2019). Los países de América Latina en los que se promocionaron los centros entre los años 2003 y 2018 son: todos desde México hasta Colombia, Chile, Perú, República Dominicana y Uruguay.

Los países y las regiones que alcanzaron mayores promedios de eficiencia y evolucionaron en términos de productividad total de los factores, presentan mejores indicadores empresariales (como la capacidad innovadora y la sofisticación del ambiente de negocios); estructurarles (como el tamaño de los mercados interno y externo y la calidad de la demanda), y sistémicos (como la oferta y la calidad de la infraestructura, la salud, la educación básica y superior, la capacitación de la mano de obra y el ambiente macroeconómico), que son importantes para que los países en desarrollo sean más competitivos (Medeiros et al., 2019).

Además, se destaca la importancia de las autoridades políticas y económicas en esos países, pues la prestación de servicios de infraestructura económica, salud y educación y el mantenimiento de un ambiente macroeconómico favorable pueden contribuir a que los países alcancen altos niveles de competitividad $y$, en consecuencia, disfruten de sus beneficios en términos de desarrollo económico y humano. Esto permitiría que las empresas se desarrollaran en un ambiente adecuado para su funcionamiento (Medeiros et al., 2019). 


\section{METODOLOGÍA}

La técnica metodológica que se emplea en este estudio, ha sido utilizada en diferentes investigaciones y se ha logrado llegar a resultados muy satisfactorios (Hernández-Sánchez et al., 2020; Sánchez-García et al., 2018). Siendo así, es de esperar que los resultados que se obtienen en esta investigación, también corresponden a información confiable y valiosa a fin de lograr los objetivos propuestos y responder las preguntas de investigación.

\section{Diseño de Estudio}

Esta investigación es cualitativa. Se emplean las fuentes secundarias para llevar a cabo una revisión sistemática de la literatura sobre Centros de Desarrollo Empresarial (SBDC) y sintetizar la literatura científica.

Se han empleado férreos mecanismos de control a fin de reducir los sesgos al mínimo, como el método PRISMA (Liberati et al., 2009; Urrútia \& Bonfill, 2010) en el proceso de elección y descarte de los artículos. También, nos hemos apoyado en un protocolo previo de criterios explícitos uniformemente aplicados a todos los artículos, a fin de acotar el tema y centrarnos en los objetivos planteados.

Se han empleado todos los documentos que se encontraban en las bases de datos, a fin de no perder información relevante que haya sido publicada en otros formatos diferentes a los artículos científicos. Lo anterior con el objetivo de reducir al máximo el riesgo de sesgo y que, a su vez, nos permitieran determinar la frecuencia y relación de los autores más co-citados del tema; la frecuencia y relación de las fuentes en que mayormente se publica sobre el tema, y el progreso en la investigación a través del tiempo. Por otro lado, se analizaron los artículos científicos que fueron evaluados por pares a fin de garantizar que información en destalle fuera más precisa y se ajustara a los estándares de calidad de extracción de la información.

Este estudio bibliométrico comprende los siguientes pasos: 1. Selección de los artículos relacionados con CDE (SBDC) 2. Aplicación de métodos estadísticos para extraer la información relevante y 3. Inclusión de una síntesis narrativa sobre los mayores hallazgos del estudio (Hernández-Sánchez et al., 2020).

La estructura de análisis de las redes bibliométricas, se basa en un enfoque para el mapeo y agrupamiento unificado (van Eck, \& Waltman, 2010), que proporciona información sobre la estructura de una red, sobre los campos de investigación, cómo se relacionan los temas entre si y cómo se desarrolló el tema a lo largo del tiempo. De manera que a través de este análisis cuantitativo y cualitativo de la evidencia encontrada se logra responder a las preguntas de investigación.

La búsqueda de artículos, procedimientos y revisiones relevantes se llevó a cabo en las siguientes tres bases de datos: the Social Science Citation Index (SSCI), Scopus y EBSCOhost, sin restricciones temporales. Se consideran estas tres bases de datos porque SSCI es la base de datos de citas más antigua, tiene una gran cobertura con datos de citas y datos bibliográficos que datan de 1900 (Boyle \& Sherman 2006), Scopus tiene un rango diario más amplio publicado desde 1995 (Sánchez-García et al., 2018) y EBSCOhost incluye mayormente documentos latinoamericanos.

\section{Estrategia de investigación}

Se utilizaron técnicas de citación para seleccionar, analizar e interpretar los patrones de citas dentro de la literatura del SBDC y el método PRISMA (Liberati et al., 2009; Urrútia \& Bonfill, 2010). Una vez elaborado el protocolo se procede a buscar la información. 
En los buscadores de las dos bases de datos (SSCI, Scopus y EBSCOhost), se empleó el siguiente procedimiento sistemático: Se eligió la frase "Small Business Development Center*" para llevar a cabo una búsqueda booleana. Se utilizan las comillas y el asterisco a fin de que el buscador encontrará todas las posibles opciones de escritura.

En este primer paso, se logran identificar 37 documentos de SSCI, 41 documentos de Scopus y 55 de la base de datos EBSCOhost. La información se extrae sin ninguna restricción en el tiempo, pues se busca analizar las tendencias en la investigación, se consideran solo artículos que fueran arbitrados. Posteriormente, se comparan las extracciones para eliminar los artículos duplicados en la misma base y entre las bases de datos. Se revisa el "abstract" de cada artículo, para verificar que todos los artículos seleccionados coincidieran con el tema de investigación (Hernández-Sánchez et al., 2020; Sánchez-García et al., 2018).

De esa manera, se excluyen 2 artículos duplicados en la misma base, 46 duplicados entre bases y no se encuentran artículos cuya información no se relacionará directamente con los SBDC. En total, con el programa VOSViewer (van Eck, \& Waltman, 2010) y Excel, se analizan 84 artículos. Por tanto, la calidad de la información fue óptima y permitió responder de manera asertiva las preguntas de investigación.

\section{Diagrama de flujo de los estudios recuperados para la revisión}

En el cuadro 1 se detalla: la identificación, la extracción sistematizada de los artículos, los códigos boléanos que se emplearon y la cantidad de artículos que se obtuvieron de cada una de las relaciones. Con el propósito de ser exhaustivo en la búsqueda y ver el efecto a través del tiempo, no se restringió por año. Además, se empleó en el filtro el "título" a fin de ser precisos con la información que se buscaba y por la cantidad de reportajes, notas y publicaciones parcializadas, se consideró solamente artículos que fueran arbitrados por pares, a fin de reducir el sesgo asociado (Hernández-Sánchez et al., 2020; Sánchez-García et al., 2018).

En la parte de elección, se indica la cantidad de artículos que se excluye por estar duplicados en la misma base de datos al realizar diferentes búsquedas. Posteriormente, al unir las bases de datos, se eliminan los artículos duplicados entre las bases de datos, u otros documentos, que al hacer la revisión del título no estuvieran relacionados con el tema. Finalmente, se indica la cantidad de artículos que analizan.

\section{CUADRO 1 DIAGRAMA DE FLUJO “SMALL BUSINESS DEVELOPMENT CENTER”}

\begin{tabular}{lccc}
\hline Título del artículo (revisión de par) & SSCI & Scopus & $\begin{array}{c}\text { EBSCO/ } \\
\text { host }\end{array}$ \\
\hline "Small Business Development Center*" & 37 & 41 & 14,2 \\
Total de artículos sin exclusiones & 37 & 41 & 55 \\
Menos duplicados en la misma base & 3 & \\
Menos repetidos entre las bases & 46 \\
\hline Menos documentos sin relación directa & 0 \\
Total de artículos para analizar & 84
\end{tabular}

Nota: Sigue el método Prima (Liberati et al., 2009; Urrútia \& Bonfill, 2010)

Fuente: Elaboración propia 
Fuentes de datos, secciones de estudios y extracción de datos.

La investigación se llevó a cabo utilizando el software VOSviewer versión 1.6 .7 (van Eck, \& Waltman, 2010) para construir y visualizar mapas bibliométricos a través de la técnica de similitudes, así como para identificar clústeres y sus redes de referencia (van Eck \& Waltman, 2010; Waltman et al., 2010) y se empleó Mendeley como gestor bibliográfico (Hernández- Sánchez et al., 2020).

Con VOSviewer (van Eck, \& Waltman, 2010) se pueden distinguir dos tipos de mapas bibliográficos, uno basado en la distancia y otro en los gráficos. "Los mapas basados en la distancia son mapas en los que la distancia entre dos elementos refleja la fuerza de la relación entre los elementos. Una distancia menor generalmente indica una relación más fuerte" (van Eck, \& Waltman, 2010), lo que facilita la identificación de grupos de elementos relacionados. Los basados en los gráficos, las líneas se dibujan entre los elementos para indicar las relaciones. Para este estudio, utilizamos mayormente los mapas basados en distancia.

\section{Análisis de datos}

Para el análisis de los datos se plantean dos estrategias. La primera, corresponde al análisis de los clústeres obtenidos con el programa Vosviewer y la segunda, corresponde al análisis bibliométrico de la información. De esta manera, hemos podido estudiar el tema dentro del campo académico y percibir el avance en la investigación desde 1977 hasta el 2019.

\section{Análisis de Clúster}

Para identificar todos los posibles campos de investigación y las variables que se han asociado a los "SBDC", se llevó a cabo un análisis de co-ocurrencia con un mínimo de 2 veces de ocurrencia por palabra, para un total de frecuencia de 57 palabras claves agrupadas en 7 cluster.

De ese modo las variables son agrupadas por el sistema como se observa en el siguiente universo de palabras claves (Figura 1), donde se pueden observar la agrupación alrededor de los principales temas tratados en las investigaciones, por ejemplo: desarrollo de negocios; modelos educativos; capital de alto riesgo; exportación; administración de negocios, educación de negocios; autoempleo, entre otros.

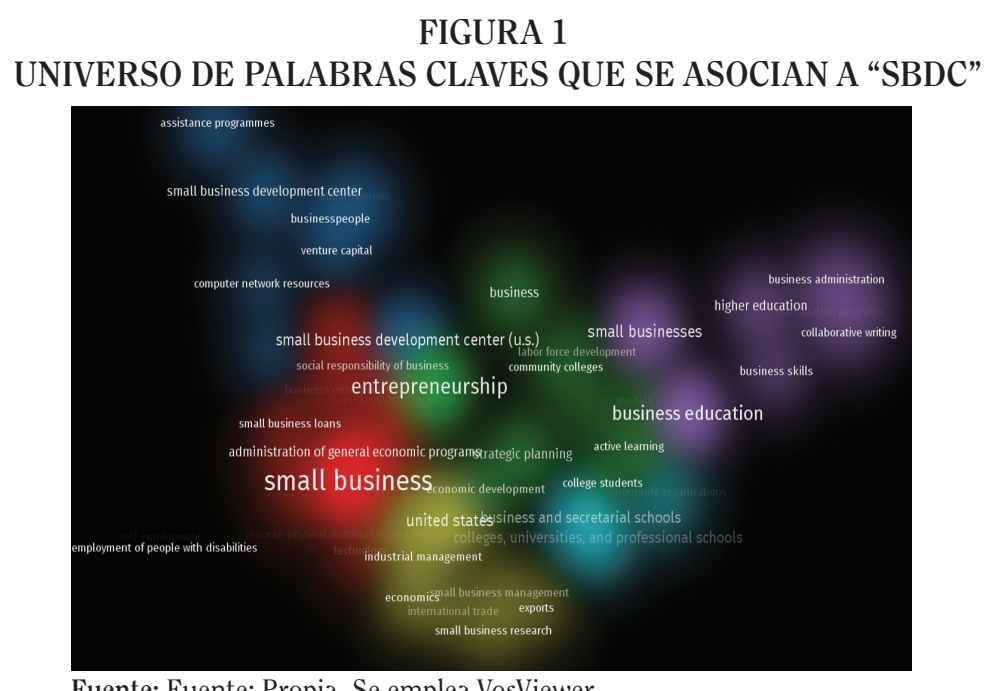

Fuente: Fuente: Propia. Se emplea VosViewer. 
Posteriormente para identificar los campos de investigación más relevantes, llevamos a cabo un análisis de co-ocurrencia para determinar la mayor frecuencia en el uso de las variables asociadas, de esa manera, se considera un mínimo de 5 veces de ocurrencia por palabra, para un total de frecuencia de 8 palabras claves agrupadas en 2 clúster.

Las variables agrupadas por el sistema se relacionan en dos grandes cluster, uno relacionado con los negocios (el de color rojo) y el otro en torno a la educación (el de color verde), como se observa en la figura 2.

\section{FIGURA 2 \\ DOS CLÚSTERES PRINCIPALES ASOCIADOS A LAS “SBDC”}

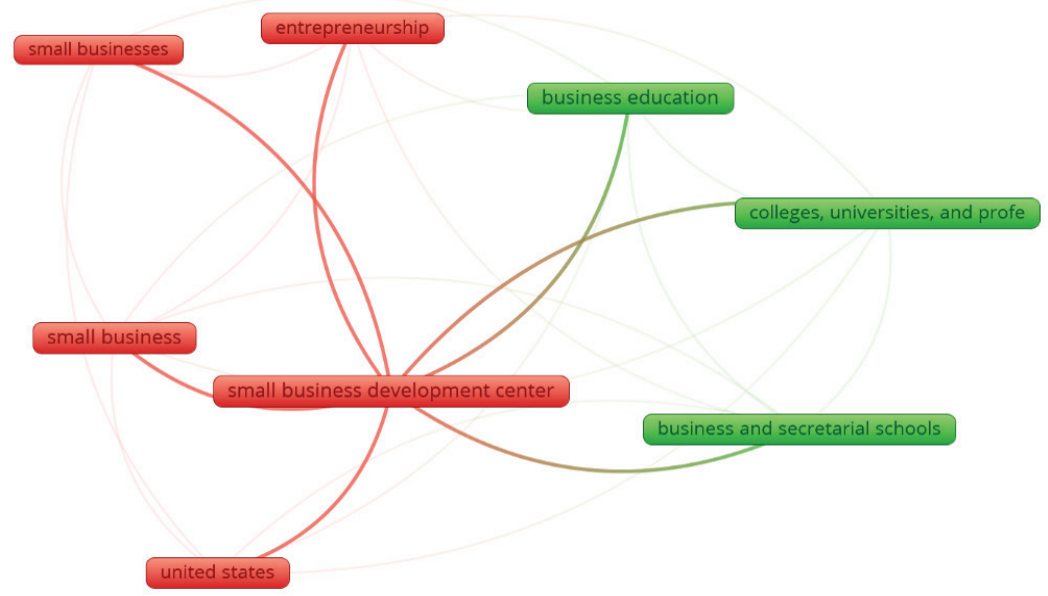

Fuente: Propia. Se emplea VosViewer.

El mapeo y agrupamiento combinado que se muestra en la figura 2, proporciona una visión general de la estructura en el campo de investigación de "SBDC". Cada clúster está representado por un color diferente que exhibe su importancia relativa, la proximidad y relación entre ellos -basado en medida de similitud conocida como fuerza de asociación. Es decir, "cuanto mayor sea el número de elementos vecinos y cuanto menor sea la distancia entre estos elementos y el punto de interés, mayor será la consistencia e importancia del área de investigación" (van Eck, \& Waltman, 2010).

La similitud entre los ítems es proporcional a la relación entre ellos, de manera que en la figura 2, se pueden distinguir claramente los dos clústeres asociados al núcleo central que es "Small Business Development Center". A la derecha se encuentra el primer clúster en verde, relacionado con la educación y a la izquierda en rojo el clúster relacionado con los negocios y su desarrollo. A continuación, los estudiaremos más a profundidad.

Clúster de color verde. Este clúster se encuentra asociado con información relacionada a la educación. El mismo recaba documentos científicos que muestran que muchas veces los SBDC, apoyan a las empresas a lograr sus objetivos de desarrollo, a través de la participación activa de las universidades. Esto por cuanto las universidades se ha convertido en las últimas décadas en entes activos del desarrollo económico local, pues sus políticas se logran enlazar con las de SBDC, de manera que logran un impacto más significativo en el trabajo local (Steinnes, 1987).

Algunos ejemplos de esta coalición que va en beneficio de las empresas, se detallan seguidamente: los programas de educación empresarial en el que la experiencia tradicional de los graduados se combina con programas profesionales de desarrollo ejecutivo, permiten lograr el desarrollo de habilidades en consultoría al compartir experiencias con consultores (Roebuck \& 
Brawley, 1996). Otro ejemplo es el personal de la biblioteca de la Universidad Central del Estado de Missouri y del personal del SBDC, que han ayudado a los desarrolladores de nuevos productos en la búsqueda de patentes y marcas registradas, y tecnología e investigación de mercado, de manera que, muchos clientes de esta asociación han traído con éxito productos y servicios al mercado (Medaris \& Manley, 1997).

Asimismo, la Universidad Internacional de Florida (FIU) se convirtió en la casa del SBDC y el Instituto de Eventos Extremos (EEI). Ambos institutos han colaborado para desarrollar y difundir conocimiento, procesos y mejores prácticas a través de varias actividades y canales como la consultoría empresarial y la elaboración del plan de continuidad comercial, pasantías y el equipo de herramientas de resiliencia ante desastres para pequeñas y medianas empresas (Sarmiento et al., 2016).

Las empresas y los inventores requieren información oportuna y completa sobre los nuevos productos que están desarrollando y los SBDC tienen el propósito es ayudar las pequeñas empresas locales, inventores y desarrolladores de nuevos productos a transformar sus ideas en productos comercializables, lo que ha permitido hacer un equipo multidisciplinario con resultados notables a nivel educativo y de industria (Politis \& Shooter, 2001), como es el caso del SBDC de la Universidad de Bucknell que trabaja en estrecha colaboración con los estudiantes de la facultad de ingeniería.

Este efecto de colaboración no solo se ha desarrollado en América, sino que tras el colapso de los gobiernos socialistas de Europa del Este, Estados Unidos ayudó a esos países en la transición a una economía de libre mercado, como es el caso de Rumania, donde se brindó asistencia a las universidades para revisar el currículo y la enseñanza de la pedagogía y para establecer SBDC que prestaran sus servicios en el mantenimiento de programas de asistencia para pequeñas empresas (Oprea \& Bernier, 2004).

Los mecanismos para aumentar la transferencia de tecnología entre las universidades y la industria han proliferado rápidamente a medida que las instituciones de educación superior se han vuelto mucho más emprendedoras y han acogido los SBDC-, por lo que la gestión universitaria y la política gubernamental (Dill, 1995) deben ser consideradas en este proceso, $y$ dado que no siempre es posible transferir los beneficios derivados de las nuevas tecnologías a los aspirantes a empresarios $y$ propietarios de pequeñas empresas por medio de la enseñanza tradicional, existen otras opciones como la educación móvil (M-educación) (McInturff \& Tapie Rohm, 2002) que puede contribuir de manera satisfactoria.

Los SBDC también, pueden ser un medio para la realización de diferentes estudios científicos que les facilitan a las empresas la toma de decisiones, como en el caso de las empresas familiares y la participación de los miembros (Werbel \& Danes, 2010); o el impacto relativo de la asistencia de asesoramiento externo y los cursos de emprendimiento en la creación y el desempeño de nuevas empresas (Chrisman et al., 2012); o las empresas familiares que se benefician más de las reservas de recursos basadas en relaciones externas, mientras que las empresas no familiares se benefician más de las reservas de recursos basadas en habilidades funcionales (Chrisman et al., 2009).

Finalmente, en este clúster, encontramos análisis que buscan determinar el impacto de la educación empresarial o el asesoramiento tuvieron en el largo plazo en el crecimiento de nuevas empresas. Los resultados indican que el asesoramiento tiene un impacto significativo en el rendimiento de la empresa, no así los cursos de emprendimiento. Los cursos de emprendimiento parecen influir indirectamente en el rendimiento de nuevas empresas al aumentar las probabilidades de inicio. En contraste, los cursos de emprendimiento están relacionados con la creación de empresas, mientras que el asesoramiento no lo está (Chrisman et al., 2012). 
Clúster de color rojo. Este clúster analiza el impacto que los SBDC tuvieron en los negocios. Es posible que mucho del crecimiento acelerado de la actividad empresarial (Sánchez-García et al., 2018) se encuentre relacionada con los esfuerzos conjuntos que realizaron con los gobiernos.

Por ejemplo, los programas SBDC realizaron actividades de asesoramiento para los individuos o grupos con intención emprendedora $y$ aunque el dato no fue preciso al atribuir la causa a los efectos en los negocios dinámicos, este estudio indica que se experimentó una taza mayor de lo esperado, en el inicio de negocios y de supervivencia, lo que se traduce también en creación de nuevos puestos de trabajo, aumento de ventas y nuevos ingresos fiscales generados por las empresas clientes que excedieron el costo de la prestación de los servicios (Chrisman et al., 1987).

Unos años después del estudio anterior, se logra comprobar que el impacto económico de las actividades de asesoramiento del SBDC mejora el rendimiento de los clientes a largo plazo pues logran generar mayores ingresos por nuevas ventas, así como la apertura de empleos. La mejora en el rendimiento se da a razón de $\$ 2.61$ de ingreso fiscal por cada dólar gastado en programas de SBDC (Chrisman \& Katrishen, 1994, 1995).

También se logra probar que la asistencia estratégica provista por el SBDC se asoció de manera significativa y positiva con el valor percibido de sus servicios. Caso contrario ocurrió con la asistencia operativa y la administrativa, pues los clientes percibieron la asistencia estratégica como más valiosa que las otras dos (Chrisman, 1989).

En cuanto a los estudios de mujeres emprendedoras, los resultados indicaron que los clientes masculinos y femeninos del SBDC son prácticamente idénticos en términos de sus necesidades de asistencia y están igualmente satisfechos con la asistencia recibida. Los SBDC son igualmente accesibles para ambos géneros (Chrisman et al., 1990).

Por su lado, los centros de innovación, las incubadoras y los SBDC tienen su lugar en el desarrollo económico. Este clúster también incluye un estudio sobre la importancia de los ecosistemas empresariales y considera importante dentro de este ecosistema el apoyo de las organizaciones híbridas, como incubadoras, aceleradores y SBDC (Roundy, 2017).

Además, enfatiza ayudar a los empresarios tecnológicos, principalmente por su aporte en número de empleos (Udell, 1988), pero ¿Qué se necesita para que una región fomente el emprendimiento tecnológico? Si se espera que el capital de riesgo produzca una riqueza extraordinaria, debe estar acompañado por el acceso a ideas novedosas, modelos a seguir, foros informales, oportunidades específicas de la región, redes de seguridad, acceso a grandes mercados y liderazgo ejecutivo (Venkataraman, 2004).

Los estudios asociados a este clúster identifican importantes implicaciones que también actualmente sería importante considerar, por ejemplo los empresarios con recursos limitados pueden obtener asistencia comercial efectiva de la SBDC de forma gratuita, $y$ los beneficios para la sociedad derivados de este servicio superan con creces el costo de proporcionarlos (Chrisman et al., 1987).

Al formular las políticas públicas es importante considerar que existe poca evidencia para garantizar un tratamiento especial o programas especiales para las mujeres, pues los resultados sugieren que sería mejor si los estudios futuros se concentraran en comportamientos y factores estratégicos que afectan el desempeño de nuevas empresas (ya sean propiedad de hombres o mujeres), en lugar de simplemente comparar las características de los empresarios (Chrisman et al., 1990)

Finalmente, un último estudio señala que los directores de los programas SBDC deben comprender las capacidades de su personal y las necesidades de sus clientes para poder formular una estrategia de consultoría efectiva a fin de alcanzar la mayor proyección y por tanto beneficio económico y social (Chrisman, 1989). Un punto importante a considerar es el uso de las páginas web de los SBDC, pues los contenidos deben ajustarse a las necesidades del entorno (Phillips, 2014) facilitar la comunicación. 


\section{Revisión Bibliométrica}

La siguiente información bibliométrica deriva del análisis y suma de frecuencias de las publicaciones bajo estudio por año. Como se observa en la figura 3, es posible inferir la importancia que tiene cada perspectiva de acuerdo con la frecuencia de publicación en el tiempo. Al realizar una comparación del avance en la investigación desde 1977 hasta el 2019 asociada a los dos clústeres principales, se puede visualizar el crecimiento de las investigaciones en dichas áreas.

Siendo así, se identifica que la línea roja, que representa la tendencia de las investigaciones que se enfocan mayormente en la afectación de los SBDC en los negocios, crece durante todo el periodo de publicaciones. Sin embargo, a partir del 2009 decrece. Por otro lado, la línea verde, que relaciona los SBDC con la educación, aunque crece en menor medida que la línea de gestión en los negocios, continua su crecimiento en el último periodo. Por lo que se considera, que la perspectiva a la que los investigadores le han otorgado mayor importancia en la última década y de ahí el aumento en las publicaciones, es la relacionada con educación empresarial.

\section{FIGURA 3 \\ DOS CLÚSTERES PRINCIPALES ASOCIADOS A LAS “SBDC”}

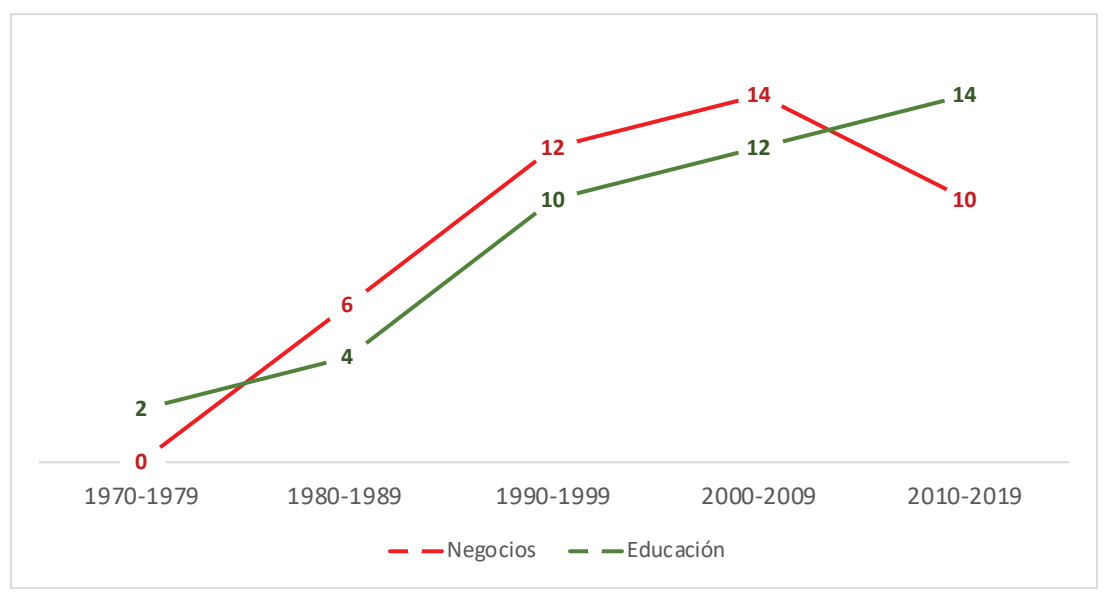

Fuente: Propia.

Con respecto a los mayores exponentes sobre SBDC, es decir aquellos que fueron citados por lo menos 5 veces, se mencionan principalmente 21 autores agrupados en 4 clúster como se observa en la figura 4. Entre ellos se pueden mencionar con más enlaces y relaciones más fuertes a Chrisman, Danes, Thrall, Chua y Sharma. 


\section{FIGURA 4 \\ CLÚSTERES DE CITACIÓN DE AUTORES}

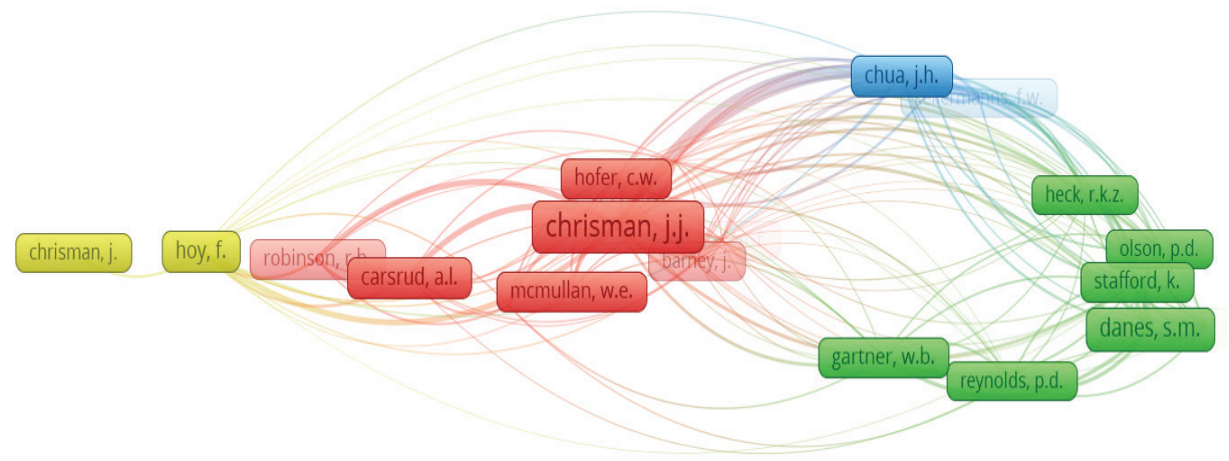

Fuente: Propia. Se emplea VosViewer.

Finalmente, respondiendo al último objetivo sobre aquellas fuentes en que se publica más sobre los SBDC, con un número mínimo de cocitación en fuentes de publicación de 10, se tienen las siguientes revistas agrupados en dos cluster, como se observa en la figura 5.

\section{FIGURA 5 \\ CLÚSTERES DE FUENTES DE PUBLICACIÓN}

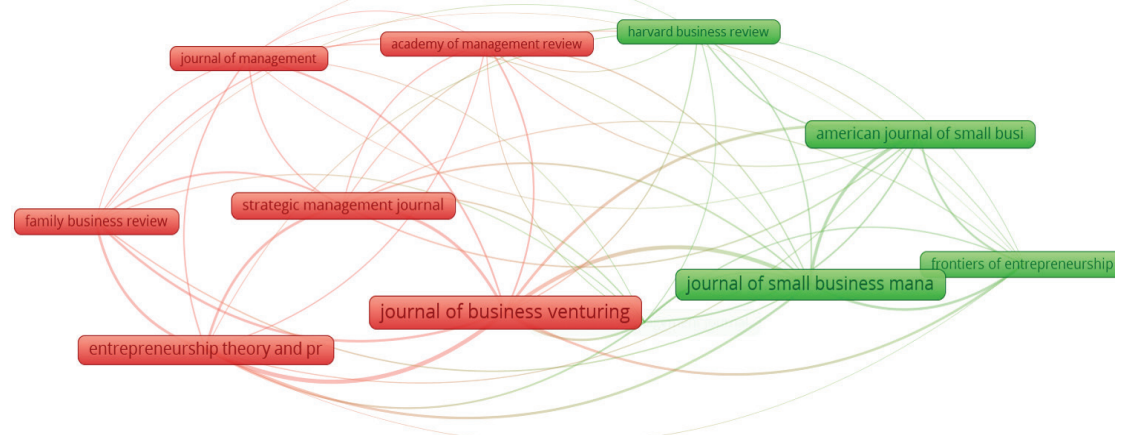

Fuente: Propia. Se emplea VosViewer.

Es interesante observar como los artículos que más son citados para llevar a cabo las investigaciones se encuentran publicados en revistas que se encuentran en los principales cuartiles del índice de la Journal Citation Reports. Las cinco revistas con más citaciones y fuerza en los enlaces son Journal of Business Venturing; Journal of Small Business Management; Entrepreneurship theory and practice; Strategic Management Journal y American Journal of Small Business.

\section{RESULTADOS}

\section{Síntesis de hallazgos}

La selección de los artículos se da a partir de la frase "SBDC". Se eliminaron todos aquellos estudios que fueran irrelevantes, repetidos y cuyas palabras claves no estuvieran dentro del resumen, palabras claves o título (abstract, keywords o title). El estudio de 84 artículos se realiza 
empleando dos estrategias de análisis: en la primera se analizan los clúster, se conocen todas las perspectivas relacionadas los SBDC; se expone la conformación de los clúster y se informa sobre los temas relevanes. En la segunda, se realiza un estudio bibliométrico en el que se describen los indicadores: los años en que se ha publicado y su crecimiento, la citación de los autores y en dónde se publica.

Del analisis general, es importante mencionar que los temas con los que se relaciona el termino "SBDC" son principalmente dos: los negocios y la educación. Adicionalmente, es relevante indicar como se observa en la figura 3 que la investigación en SBDC y su influencia en los negocios y la educación, han crecido considerablemente, especialmente desde 1980 cuando el gobierno de los Estados Unidos apoya la iniciativa y procura la expanción en diferentes Estados y posteriormente en América Latina.

También es importante mencionar que las investigaciones relacionadas con los SBDC, sustentan sus teorías en documentos científicos publicados en revistas de gran rigurosidad garantizando la confiabilidad de los mismos y de la información obtenida, así como también los investigadores que han aportado sus conocimientos.

Finalmente, es importante resaltar las buenas prácticas, así como los vacíos de la literatura que se encontraron. Entre las buenas prácticas se encuentran el seguimiento continuo por parte de los SBDC a las actividades de las empresas, su interés por el alcance de los objetivos y la posibilidad de potencializar la actividad en un menor tiempo, así como las asesorías que brindan.

Entre las área de estudio que será importante considerar para investigar, especialmente en América Latina, se encuentran: implementación de microcréditos que eviten el ahogamiento de las empresas pero que a la vez les permita crecimiento; asesoría estratégica en el desarrollo de negocios a través de plataformas digitales; rediseño de estrategias y replanteamiento de los negocios; aprovechamiento de las capacidades blandas; $y$ en esta época de pandemia (Covid-19) aspectos como la solidaridad podrían causar efectos positivos en la reactivación económica.

Además, los SBDC deben considerar dentro de su estructura de atención de negocios a las nuevas generaciones, su manera de percibir el entorno que los rodea, la exposición a gran cantidad de información a través de internet que les permite ser autosuficientes, controlar tiempo y espacio de sus actividades.

\section{Riesgo de sesgo}

Nuestra investigación recolecta la información existente en materia de "SBDC" de SSCI, Scopus y EBSCOhost. Estas tres bases de datos emplean criterios adecuados de selección de artículos en función de la calidad y relevancia académica. De manera que, la evaluación individual sobre el método que se empleó en cada investigación para determinar la integridad del estudio, no aplica por cuanto se considera que ese tipo de riesgo fue eliminado al emplear dichas bases de datos. Por otro lado, una vez que inició la extracción de la información, la investigación se ciñe al protocolo y a los objetivos establecidos, apartando los criterios personales que pudieran entorpecer la investigación. Siendo así, en el análisis de clúster, se establecen las relaciones entre los artículos de acuerdo con los mapas bibliográficos basados en la distancia y en la segunda parte, se analizaron otros aspectos como es la citación, fuentes y años de publicación.

\section{DISCUSIÓN}

El objetivo principal de los SBDC es proporcionar asesoría profesional a las empresas de manera que, estas se puedan desarrollar de manera exitosa ante los desafíos del ambiente (Dini, 2019). Para tal fin se ha motivado a instituciones públicas a promocionar los centros y fomentar el 
desarrollo de los mismos, con la esperanza de que las empresas puedan alcanzar niveles mayores de eficiencia y evolucionen en términos de productividad (Medeiros et al., 2019).

$\mathrm{Al}$ respecto se encontró en esta investigación que los estudios realizados sobre los SBDC principalmente están relacionados con dos perspectivas: la de educación y la de los negocios. En Estados Unidos, la mayoría de los SBDC se desempeñan a través de las universidades, por lo que la educación se convierte en un aliado de este proceso. Por su lado en América Latina, los entes involucrados son las instituciones públicas, organizaciones sin fines de lucro, agencias de desarrollo $y$ en algunos casos las universidades (Dini, 2019).

La participación de los SBDC gestionados a través de instituciones de educación ha demostrado que tiene un mayor impacto, pues permite brindar el acompañamiento que las empresas requieren, de manera que, es posible que reciban asesoramiento para mejorar el rendimiento o que se impartan cursos de emprendimiento a fin de lograr la creación de empresas (Chrisman et al., 2012). Lo cierto es que tanto los cursos de emprendimiento como el asesoramiento apoyan la creación y desarrollo de las empresas.

Asociada al clúster de los negocios, se logran identificar investigaciones que comprueban que el impacto económico de las actividades de asesoramiento mejora el rendimiento de los clientes a largo plazo (Chrisman \& Katrishen, 1994, 1995); que la asistencia estratégica se asocia con el valor percibido de los servicios (Chrisman, 1989); que las necesidades de asistencia técnica no discriminan por género (Chrisman et al., 1990); que el apoyo tecnológico a empresarios debe ser primordial (Venkataraman, 2004); y que son importantes los ecosistemas empresariales (Roundy, 2017) a fin de contribuir con el desarrollo empresarial.

Incluso cuando los programas de asesoramiento y enseñanza empresarial deban ser ajustados a cada entorno y ya sea que la iniciativa sea a través de políticas públicas o por interés de las universidades u otros entes, los SBDC adquieren importancia en el tanto buscan el beneficio paulatino y sostenido de las empresas, de manera estructurada y bajo el cumplimiento de metas específicas que permitan controlar y fomentar el avance.

\section{CONCLUSIONES Y LÍNEAS FUTURAS DE INVESTIGACIÓN}

El propósito de esta investigación fue estudiar científicamente la actividad y los alcances de los Centros para el Desarrollo de la Pequeña Empresa (SBDC). Para lograr el objetivo general se realiza una revisión sistemática de la literatura en la que no se estableció un tiempo determinado, sino que se recaba toda la información existente de las tres principales bases de datos -Scopus y SSCI y EBSCOhost-.

De conformidad con los objetivos propuestos se concluye los siguiente:

- Se logran identificar las principales perspectivas teóricas que caracterizan y definen la literatura relacionada con los SBDC: la educación y los negocios.

- Se determina que la perspectiva a la que los investigadores le han otorgado mayor importancia, especialmente en la última década, de acuerdo con un análisis de tendencias de las publicaciones, es la que se encuentra relacionada con la educación empresarial. Este análisis de tendencias se realiza desde 1977 hasta el 2019, años en los que fue posible recabar información de las bases de datos.

- Se identifican los mayores exponentes del tema a través del número de citas en los artículos asociados con los SBDC, ellos son: Chrisman, Danes, Thrall, Chua y Sharma.

- Se identifican las fuentes que mayormente son consultadas sobre los SBDC, estas son: Journal of Business Venturing; Journal of Small Business Management; Entrepreneurship theory and practice; Strategic Management Journal y American Journal of Small Business. Todas estas revistas se encuentran en los principales cuartiles del índice JCR, lo que nos confirma que la información relacionada y hasta ahora publicada ha pasado por revisión de pares. 
En cuanto a los requerimientos de estudio es decir, sobre que no se ha estudiado o es escaso el estudio, las líneas futuras de investigación deben redirigirse tanto hacia la implementación de los negocios a través de plataformas digitales, buscar microcréditos que eviten el ahogamiento de las empresas por intereses; rediseño de estrategias de cocreación; aprovechamiento de los recursos y capacidades blandas; $y$ en esta época de pandemia aspectos como la solidaridad podrían causar efectos positivos en la reactivación económica.

Además, sería importante considerar para nuevas investigaciones, cómo lograr un buen acoplamiento de los SBDC con los negocios de las nuevas generaciones, pues al haber nacido en la era de la tecnología digital y acceso a gran cantidad de información a través de internet, muchos de ellos son autosuficientes, capaces de desarrollar actividades fuera de un espacio físico definido o tiempo determinado, lo que hace que también los negocios tomen un giro hacia plataformas digitales.

\section{LIMITACIONES}

Como ocurre de manera inevitable, la investigación presenta algunas limitaciones que se encuentran asociadas a los riesgos de sesgo. El principal riesgo que visualizamos, es que no se hayan incluido todos los artículos relacionados con el tema, aun a pesar de que se incluyen tres bases de datos, sin embargo, la medida anterior puede mejorar el alcance de la muestra, pero también puede agregar artículos irrelevantes y hacer que la muestra sea cada vez más desafiante desde la perspectiva del tratamiento práctico. Adicionalmente, para subsanar el sesgo se analizaron los SBDC sin restricciones en las áreas de conocimiento y sin restricciones de tiempo.

\section{CONFLICTO DE INTERESES}

Se declara que la investigación se realizó sin relaciones comerciales o financieras que puedan interpretarse como un posible conflicto de intereses.

\section{AGRADECIMIENTOS}

Agradezco a la Universidad Estatal a Distancia en Costa Rica a través de la Vicerrectoría de Investigación por el apoyo y seguimiento de todas las actividades.

\section{REFERENCIAS}

Bosma, N., \& Kelley, D. (2019). Global Entrepreneurship Monitor 2018-19 (Vol. 8).https://www. gemconsortium.org/report/gem-2018-2019-global-report

Boyle, F., \& Sherman, D. (2006). "ScopusTM: The Product and Its Development." The Serials Librarian, 49(3): 147-53. https://doi.org/10.1300/J123v49n03_12

Chrisman, J. J. (1989). Strategic, administrative, and operating assistance: The value of outside consulting to pre-venture entrepreneurs. Journal of Business Venturing, 4(6), 401-418. https://doi.org/10.1016/0883-9026(89)90010-4

Chrisman, J. J., Carsrud, A. L., DeCastro, J., \& Herron, L. (1990). A comparison of assistance needs of male and female pre-venture entrepreneurs. Journal of Business Venturing, 5(4), 235-248. https://doi.org/10.1016/0883-9026(90)90019-P 
Chrisman J. J., Chua J. H., \& Kellermanns, F. (2009). Priorities, resource stocks, and performance in family and nonfamily firms. Entrepreneurship: Theory and Practice, 33(3), 739-760. https:// doi.org/10.1111/j.1540-6520.2009.00324.x

Chrisman, J. J., Hoy, F., \& Robinson, R. B. (1987). New venture development: The costs and benefits of public sector assistance. Journal of Business Venturing, 2(4), 315-328. https://doi. org/10.1016/0883-9026(87)90024-3

Chrisman, J. J., \& Katrishen, F. (1994). The economic impact of small business development center counseling activities in the United States: 1990-1991. Journal of Business Venturing, 9(4), 271-280. https://doi.org/10.1016/0883-9026(94)90008-6

Chrisman, J. J., \& Katrishen, F. (1995). The small business development center programme in the USA: A statistical analysis of its impact on economic development. Entrepreneurship \& Regional Development, 7(2), 143-155. https://doi.org/10.1080/08985629500000009

Chrisman, J. J., McMullan, W. E., Kirk Ring, J., \& Holt, D. T. (2012). Counseling assistance, entrepreneurship education, and new venture performance. Journal of Entrepreneurship and Public Policy, 1(1), 63-83. https://doi.org/10.1108/20452101211208362

Dill, D. D. (1995). University-industry entrepreneurship: The organization and management of American university technology transfer units. Higher Education, 29(4), 369-384. https://doi. org/10.1007/BF01383958

Dini, M. (2019). Centros de desarrollo empresarial en América Latina: Lecciones de política de cinco experiencias institucionales. https://repositorio.cepal.org/handle/11362/44589

Dini, M., \& Stumpo, G. (2018). Mipymes en América Latina. Un frágil desempeño y nuevos desafíos para las políticas de fomento. CEPAL. https://repositorio.cepal.org/handle/11362/44148.1

Hernández-Sánchez, B., Vargas-Morúa, G., González Cedeño, G., \& Sánchez-García, J. C. (2020). Discapacidad intelectual y el uso de las tecnologías de la información y comunicación: revisión sistemática. International Journal of Developmental and Educational Psychology INFAD, 2(1), 177-188. https://doi.org/10.17060/ijodaep.2020.n1.v2.1830

Liberati, A., Altman, D. G., Tetzlaff, J., Mulrow, C., Gøtzsche, P. C., Ioannidis, J. P. A., Clarke, M. Devereaux, P. J., Kleijnen, J., \& Moher, D. (2009). The PRISMA statement for reporting systematic reviews and meta-analyses of studies that evaluate health care interventions: explanation and elaboration. Journal of Clinical Epidemiology, 62(10), E10-E34. https://doi. org/10.1016/j.jclinepi.2009.06.006

McInturff, P., \& Tapie Rohm, C. E.. (2002). Developing M-education for entrepreneurs and small businesses. Journal of Internet Commerce, 1(3), 49-65. https://doi.org/10.1300/ J179v01n03_05

Medaris, L., \& Manley, M. (1997). Building a better mousetrap: Networking with community business resources. The Reference Librarian, 27(58), 41-48. https://doi.org/10.1300/ J120v27n58_05

Medeiros, V., Godoi, L. G., \& Teixeira, E. C. (2019). La competitividad y sus factores determinantes: Un análisis sistémico para países en desarrollo. Revista de la CEPAL, 2019(129), 7-27. https:// doi.org/10.18356/9c2a7060-es

Oprea, D., \& Bernier, R. E. (2004). Sustaining free market economic education in a transition economy: A romanian experience. International Journal of Public Administration, 27(11-12), 917-929. https://doi.org/10.1081/PAD-200037368

Phillips, R. L. (2014). An Analysis of Small Business Assistance Websites: Content and Delivery. Journal of Business and Finance Librarianship, 19(2), 125-141. https://doi.org/10.1080/08963 568.2014.883880

Politis, J. L., \& Shooter, S. B. (2001). Enhancing Engineering Education: The Bucknell Product Development Center. Industry and Higher Education, 15(5), 341-347. https://doi. org/10.5367/000000001101295876 
Roebuck, D. B., \& Brawley, D. E. (1996). Forging Links Between the Academic and Business Communities. Journal of Education for Business, 71(3), 125-128. https://doi.org/10.1080/088 32323.1996.10116771

Roundy, P. T. (2017). Hybrid organizations and the logics of entrepreneurial ecosystems. International Entrepreneurship and Management Journal, 13(4), 1221-1237. https://doi. org/10.1007/s11365-017-0452-9

Sánchez-García, J., Vargas-Morúa, G., \& Hernández-Sánchez, B. (2018). Entrepreneurs’ Well-Being: A Bibliometric Review. Frontiers in Psychology, 9(September), 1-19. https://doi.org/10.3389/ fpsyg.2018.01696

Sarmiento, J. P., Hoberman, G., Jerath, M., \& Ferreira Jordao, G. (2016). Disaster risk management and business education: the case of small and medium enterprises. AD-Minister, 28, 73-90. https://doi.org/10.17230/ad-minister.28.4

Steinnes, D. N. (1987). On Understanding and Evaluating the University's Evolving Economic Development Policy. Economic Development Quarterly, 1(3), 214-225. https://doi.org/10.1177/089124248700100304

Udell, G. G. (1988). Strategies for stimulating home-grown technology-based economic development. Business Horizons, 31(6), 60-64. https://doi.org/10.1016/0007-6813(88)90026-2

Urrútia, G., \& Bonfill, X. (2010). Declaración PRISMA: una propuesta para mejorar la publicación de revisiones sistemáticas y metaanálisis. Medicina Clinica, 135(11), 507-511. https://doi. org/10.1016/j.medcli.2010.01.015

van Eck, N. J., \& Waltman, L. (2010). Software survey: VOSviewer, a computer program for bibliometric mapping. Scientometrics, 84(2), 523-538. https://doi.org/10.1007/ s11192-009-0146-3

Venkataraman, S. (2004). Regional transformation through technological entrepreneurship. Journal of Business Venturing, 19(1), 153-167. https://doi.org/10.1016/j.jbusvent.2003.04.001

Waltman, L., van Eck, N. J., \& Noyons, E. C.M. (2010). A unified approach to mapping and clustering of bibliometric networks. Journal of Informetrics, 4(4), 629-635. https://doi.org/10.1016/j. joi.2010.07.002

Werbel, J. D., \& Danes, S. M. (2010). Work family conflict in new business ventures: The moderating effects of spousal commitment to the new business venture. Journal of Small Business Management, 48(3), 421-440. https://doi.org/10.1111/j.1540-627X.2010.00301.x

Este artículo se encuentra disponible mediante la licencia Creative Commons Reconocimiento-NoComercial-SinObraDerivada 3.0 Costa Rica. Para mayor información escribir a revista.iice@ucr.ac.cr. 